\title{
Biodiesel production from castor oil: ANN modeling and kinetic parameter estimation
}

\author{
Atiya Banerjee $^{1} \cdot$ Devyani Varshney $^{1} \cdot$ Surendra Kumar $^{1} \cdot$ Payal Chaudhary $^{2}$. \\ V. K. Gupta ${ }^{3}$
}

Received: 3 February 2015/Accepted: 22 April 2017/Published online: 18 May 2017

(c) The Author(s) 2017. This article is an open access publication

\begin{abstract}
This research work concerns with the transesterification of castor oil with methanol to form biodiesel. As the free fatty acid content in castor oil is more than $1 \%$, an acid catalyst namely, $\mathrm{H}_{2} \mathrm{SO}_{4}$ has been used for esterification. The experimental conditions were determined using central composite design method and the experiments were conducted in a $2 \mathrm{~L}$ working volume fully controlled reactor. The input conditions namely, catalyst concentration, methanol to oil molar ratio and temperature were varied, and $\%$ fatty acid methyl ester (FAME) content was determined. Based upon the experimental data, an ANN model has been developed which is used to predict $\%$ FAME yield for a given set of input conditions. The experimental data and the data predicted by the ANN model have been used to estimate the rate constants of a kinetic model. The ANN model predicts the \% FAME yield within $\pm 8 \%$ deviation, and the developed kinetic model shows successfully the effect of methanol to oil molar ratio on $\%$ FAME yield at $60{ }^{\circ} \mathrm{C}$ and $3 \%$ (v/v) catalyst loading.
\end{abstract}

Keywords ANN · Castor oil · Biodiesel · Parameter estimation $\cdot$ Differential equation model

Surendra Kumar

skumar.iitroorkee@gmail.com

1 Department of Chemical Engineering, Indian Institute of Technology Roorkee, Roorkee, Uttarakhand 247667, India

2 Centre for Transportation Systems, Indian Institute of Technology Roorkee, Roorkee, Uttarakhand 247667, India

3 Department of Chemistry, Indian Institute of Technology Roorkee, Roorkee, Uttarakhand 247667, India

\section{Introduction}

Global energy consumption has been steadily increasing since the beginning of the millennium and its demand is expected to rise in the coming times. Thus, the general awareness about alternative energy sources has increased manifold, which have a lower impact on the environment as a whole [1]. Sustainable sources of energy are to be explored and used to decrease the overall dependence on available primary sources of energy (fossil fuels) [2]. In this context, biodiesel appears to be a feasible option as its usage renders a reduction of above $90 \%$ in emissions of total non-combusted hydrocarbons [3]. An excellent review has been published [4] describing various vegetable oil feedstock sources used for producing biodiesel. Biodiesel is mostly produced from the edible sources such as sunflower [5], soybean [6], and rapeseed [7]. This practice is not favorable towards sustainability because of the fact that it is competitive with food and would increase the cost of both edible oil and biodiesel [8]. Therefore, non-edible oils are preferred for this purpose. Castor oil is one of the most promising nonedible sources for the production of biodiesel [9]. Conventional in situ transesterification of castor oil seeds has been performed by Hincapie et al. [10]. Several catalysts such as $\mathrm{KOH}, \mathrm{NaOH}, \mathrm{KOCH}_{3}, \mathrm{HCl}$, etc. [11] have been tried in its transesterification with ethanol. Reduction in viscosity of castor methyl and ethyl ester blends have been studied [12]. Kinetics of this process has been investigated [13-17] by varying the alcohol to oil molar ratio as well as the temperature. In one such process [18], methanol to oil ratio was varied from 50:1 to $250: 1$ and temperature from 35 to $65^{\circ} \mathrm{C}$. Optimization of castor oil transesterification reaction to produce biodiesel has been reported by many authors, but optimization of 
tranesterification reaction by the use of ANN modeling is rare in literature. It may be concluded that these processes have not been investigated in the presence of an acid catalyst from the point of view of kinetic parameter estimation. ANN modeling can be a good approach to optimize acid catalyzed reaction for biodiesel production as it is time saving, and we can optimize biodiesel production on the pilot scale with the experimental data optimized by using ANN. The FFA content in castor oil should be less than $1 \%$ for using alkaline catalyst [19]. If the limit of FFA is exceeded, then soap formation occurs which inhibits the suppression of ester [20]. Boucher et al. [21] used significant (50 wt $\%$ ) amount of $\mathrm{H}_{2} \mathrm{SO}_{4}$ associated with 10.86-93.7 methanol to FFA molar ratio for the oil-fatty acid mixture that contained 2-15\% FFAs. Heterogeneous catalyst (sulphonated polystyrene compounds) were employed by Soldi et al. [22] and 85\% conversion of fatty acid into methyl ester was reported at a high methanol to oil ratio of 100:1 and reaction time of $18 \mathrm{~h}$. In the present investigation, experiments were conducted in a lab reactor; the experimental conditions were determined by design of experiment method for methanol to oil molar ratio $=6: 1-25: 1$; catalyst amount $($ vol\% $)=1-3 ;$ temperature $\left({ }^{\circ} \mathrm{C}\right)=40-60{ }^{\circ} \mathrm{C} ; \quad$ time duration of experiments $=4 \mathrm{~h}$.

In recent years, artificial neural networks (ANNs) have been widely applied to a wide range of applications such as data prediction [23], fault detection [24], data rectification [25], process control [26], etc. It is capable of handling even incomplete data and is effective in executing fast predictions and even for non-linear generalizations [27, 28]. In 2011, performance of linear and non-linear calibration techniques were compared in predicting biodiesel properties from near IR spectra by Balabin et al. [29]. Evaluation of the intensification of biodiesel production from waste goat tallow was performed using response surface methodology (RSM) and ANN with appreciable modeling efficiency by Chakraborty et al. [30].

This study on castor oil transesterification in presence of acid catalyst aims to use ANN for modeling the experimental data obtained as decided by central composite design (CCD) and then predicting fractional formation profile of FAME at optimized conditions, determined by RSM. Further, the developed ANN model has been used for developing a kinetic model and estimating its rate constants by a suitable parameter estimation method. Broadly, the approach adopted here is to use the available experimental data for ANN model representation without imposing additional requirements on the number of process measurements. The available experimental data and those predicted using ANN model have been used to estimate the kinetic parameters.

\section{Materials and methods}

Materials and methods as discussed in this section have been described in detail in the doctoral thesis of Payal [31] which is concerned with experimental and modeling studies of castor oil transesterification.

\section{Chemicals}

Pharmaceutical grade castor oil was purchased from the local market through the authorized standard chemical supplier of the institute. All chemicals; $\mathrm{H}_{2} \mathrm{SO}_{4}(97 \%)$, anhydrous methanol, sodium bisulphate $\left(\mathrm{Na}_{2} \mathrm{SO}_{4}\right)$ were of analytical grade and were purchased from Merck India and used without further purification. Pure standard methyl esters were purchased from Sigma Aldrich, USA.

\section{Experimental setup}

A lab reactor (Applikon, Schiedam, The Netherlands, stirred type, capacity: $3 \mathrm{~L}$, ez control system) was used for conducting the reaction. The reactor was $3 \mathrm{~L}$ double jacketed borosilicate glass reactor having $2 \mathrm{~L}$ working volume. The temperature was regularly monitored by the display of the system. The reflux system was used to avoid the vaporization of methanol from the reaction system. The temperature of the reaction system was controlled by the circulation of water through outer wall of the reactor vessel. Mechanical stirring was used for the proper mixing of the reaction mixture in the reactor.

\section{Experimental procedure}

Initially $1 \mathrm{~L}$ castor oil was transferred into the reactor and heated till it reached the desired temperature. Appropriate quantities of both methanol and catalyst (sulphuric acid) were mixed thoroughly at the preset temperature separately. The above mixture of catalyst and methanol was then transferred to the reactor where the mixing was carried out at a speed equivalent to relative centrifugal force $(\mathrm{rcf})=32.2 \times \mathrm{g}$ for the preset reaction temperature and time $4 \mathrm{~h}$. The required mixing intensity $(\mathrm{rcf}=32.2 \times \mathrm{g})$ was optimized prior to conducting the experiments at constant methanol/oil molar ratio 6:1, catalyst concentration $1 \%$ and reaction temperature of $60{ }^{\circ} \mathrm{C}$. The samples were taken up to $4 \mathrm{~h}$ regularly at the $20 \mathrm{~min}$ time interval and total 12 samples were collected during this time period. The samples were collected in $15 \mathrm{~mL}$ centrifuge tubes, filled with $5 \mathrm{~mL}$ of distilled water. Shaking and quenching of the samples were done immediately, and the centrifuge tubes with the sample were kept in an ice bath immediately. The samples were washed and centrifuged at 
rcf $=894 \times g$ for $20 \mathrm{~min}$ to separate ester layer. After centrifuging the sample, two separate layers were formed; the bottom one contained the glycerol and catalyst in water phase while the upper layer is of ester.

\section{Gas chromatography analysis}

Analysis of all samples for methyl ester formation (FAME content) was carried out using the gas chromatograph (GC) (Nucon Gas Chromatograph, 5765, India), equipped with a flame ionization detector (FID) and a capillary column with dimension of $0.55 \mathrm{~mm}$ I.D $\times 10 \mathrm{~m}$ length $\times 0.50 \mathrm{~m}$ thickness. Nitrogen was used as carrier gas. The column temperature was kept at $170{ }^{\circ} \mathrm{C}$ for $1 \mathrm{~min}$, heated at $10{ }^{\circ} \mathrm{C} /$ min up to $240{ }^{\circ} \mathrm{C}$ and then it was maintained constant. The temperature of the injector and detector was set at 220 and $240{ }^{\circ} \mathrm{C}$, respectively. Methyl heptadecanoate was used as internal standard for GC. The analysis was done by injecting $1 \mu \mathrm{L}$ sample into the column. Every sample has been analyzed three times for \% FAME yield and the average of three values has been taken.

Quantitative analysis of \% ME was done using European standard EN 14103:2003. The \% ME yield (or \% FAME yield) was calculated using equation

$\%$ FAME yield $=\frac{\sum A-A_{\mathrm{EI}}}{A_{\mathrm{EI}}} \times \frac{C_{\mathrm{EI}} \times V_{\mathrm{EI}}}{m} \times 100$,

where $\sum A$ is the total peak area from methyl esters in $\mathrm{C}_{14 ; 0}-\mathrm{C}_{24 ; 1}, A_{\mathrm{EI}}$ is the peak area corresponding to methyl heptadecanoate, $C_{\mathrm{EI}}$ is the concentration of the methyl heptadecanoate solution $(\mathrm{mg} / \mathrm{mL}), V_{\mathrm{EI}}$ is the volume of methyl heptadecanoate solution $(\mathrm{mL})$ and $m$ is the mass of the sample $(\mathrm{mg})$.

\section{Experimental design}

Central composite design (CCD) is one of the most commonly used techniques for the optimization of experiments. Transesterification of castor oil with ethanol was studied by Cavalcante et al. [17] using a central composite rotatable design. Optimum reaction conditions were determined as oil/ethanol molar ratio of $1: 11$, catalyst amount of $1.75 \%$ $\mathrm{KOH}$, and reaction time of $90 \mathrm{~min}$ and $86.32 \%$ of biodiesel yield was obtained. To apply this design, the variation levels for each variable must be specified clearly. Accordingly, the variables are transformed into coded variables bearing the following relationships [32]:

$X_{j}=\left(x_{j}-x_{j}^{0}\right) / \Delta x_{j}$,

$x_{j}^{0}=\left(x_{j}^{\max }+x_{j}^{\min }\right) / 2$,

$\Delta x_{j}=\left(x_{j}^{\max }-x_{j}^{\min }\right) / 2$,

where $X_{\mathrm{j}}$ : coded value of variable, $x_{j}^{0}$ : basic level, $x_{j}$ : actual value, $\Delta x_{j}$ : level of variation.

Central composite design is a factorial design with center points, augmented with a group of axial points (also called star points). As the distance of the factorial point from the center of the design space is defined as \pm 1 unit for each factor, the distance of a star or axial point from the center of the design is $\pm \alpha$ with $(\alpha)>1$. In this study, the CCD was used to optimize three parameters (methanol/oil molar ratio, catalyst amount, and temperature) for enhancing the \% FAME yield. In CCD, the total number of experimental combination was $2^{k}+2 k+n$, where ' $k$ ' is the number of independent variables and ' $n$ ' is the number of repetitions of experiments at the central axis point to reduce the pure error [16, 33]. Totally, 20 experiments were required for this work. Here, the run numbers 18,19 , and 20 were not considered because the experiment was not feasible to be performed under these conditions as either catalyst amount or temperature was low [34] and 6 were repeated experiments. The dependent variables for this study were $\%$ FAME yield, $X_{\mathrm{c}}$ (\%) and the independent variables selected were: methanol/ oil molar ratio $\left(x_{1}\right)$, catalyst amount $\left(x_{2}\right)$, and temperature $\left(x_{3}\right)$. The range and levels of individual variable factor have been given in Table 1. The experimental data of the FAME yield for various catalyst amounts, molar ratios, and temperatures have been given in Table 2a, b. These data (columns $\mathrm{A}-\mathrm{J}$ in Table $2 \mathrm{a}, \mathrm{b}$ ) have also been used to train the neural network for the kinetic modeling of the process. Properties of the castor oil FAME produced were also measured and are given in Table $3[31,35]$. Except viscosity and water content, all other properties of the FAME were found to be satisfactory and within standard range of values [36]. Therefore, this biodiesel may be used for blending with other fuels.
Table 1 Independent variables and levels used for CCD

\begin{tabular}{lllcrr}
\hline S. no. & Variables & Coded symbols & \multicolumn{2}{l}{ Range and levels } \\
\cline { 4 - 6 } & & & -1 & 0 & +1 \\
\hline 1 & Methanol/oil molar ratio & $\mathrm{x}_{1}$ & 6 & 15.5 & 25 \\
2 & Catalyst amount (vol\%) & $\mathrm{x}_{2}$ & 1 & 2 & 3 \\
3 & Temperature $\left({ }^{\circ} \mathrm{C}\right)$ & $\mathrm{x}_{3}$ & 40 & 50 & 60 \\
\hline
\end{tabular}


Tables 2 Experimental results on $\%$ FAME formation with time

\begin{tabular}{|c|c|c|c|c|c|c|c|}
\hline \multirow[t]{2}{*}{ S. no. } & \multirow[t]{2}{*}{ Time (min) } & \multicolumn{6}{|c|}{$\%$ formation of FAME, $X_{\mathrm{c}}$} \\
\hline & & $\begin{array}{l}\text { A } \\
1 / 60 / 25: 1\end{array}$ & $\begin{array}{l}\text { B } \\
1 / 60 / 6: 1\end{array}$ & $\begin{array}{l}\mathrm{C} \\
3 / 60 / 25: 1\end{array}$ & $\begin{array}{l}\text { D } \\
\text { 3/60/6:1 }\end{array}$ & $\begin{array}{l}\mathrm{E} \\
1 / 40 / 25: 1\end{array}$ & $\begin{array}{l}\mathrm{F} \\
1 / 40 / 6: 1\end{array}$ \\
\hline \multicolumn{8}{|l|}{ (a) } \\
\hline 1 & 0 & 0 & 0 & 0 & 0 & 0 & 0 \\
\hline 2 & 20 & 7.50 & 13.81 & 31.50 & 16.88 & 5.32 & 5.95 \\
\hline 3 & 40 & 9.51 & 21.46 & 40.79 & 20.58 & 6.95 & 7.80 \\
\hline 4 & 60 & 12.96 & 24.93 & 47.06 & 24.63 & 8.45 & 9.35 \\
\hline 5 & 80 & 16.82 & 28.81 & 54.59 & 26.75 & 12.23 & 10.54 \\
\hline 6 & 100 & 21.59 & 31.92 & 59.79 & 29.04 & 13.94 & 12.09 \\
\hline 7 & 120 & 32.04 & 35.96 & 63.06 & 31.59 & 16.94 & 15.15 \\
\hline 8 & 140 & 36.90 & 39.79 & 67.05 & 37.95 & 18.45 & 18.99 \\
\hline 9 & 160 & 40.32 & 41.71 & 69.09 & 44.79 & 20.18 & 21.95 \\
\hline 10 & 180 & 46.65 & 42.05 & 71.09 & 48.09 & 22.65 & 24.65 \\
\hline 11 & 200 & 53.05 & 44.19 & 73.53 & 50.05 & 26.94 & 26.56 \\
\hline 12 & 220 & 62.18 & 46.63 & 75.59 & 52.76 & 28.42 & 28.19 \\
\hline 13 & 240 & 72.55 & 48.29 & 76.85 & 55.27 & 31.45 & 29.95 \\
\hline \multirow[t]{3}{*}{ S. no. } & Time (min) & \multicolumn{6}{|c|}{$\%$ formation of FAME, $X_{\mathrm{c}}$} \\
\hline & & G & $\mathrm{H}$ & I & $\mathrm{J}$ & $\mathrm{K}$ & \\
\hline & & 3/40/25:1 & 3/40/6:1 & 2/50/15.5:1 & $3.68 / 50 / 15.5: 1$ & 3/45/12:1 & 3/60/18:1 \\
\hline \multicolumn{8}{|l|}{ (b) } \\
\hline 1 & 0 & 0 & 0 & 0 & 0 & 0 & 0 \\
\hline 2 & 20 & 7.23 & 16.28 & 9.93 & 33.45 & 18.41 & 20.50 \\
\hline 3 & 40 & 9.59 & 19.15 & 14.56 & 38.56 & 23.95 & 34.16 \\
\hline 4 & 60 & 12.81 & 23.92 & 21.72 & 42.69 & 26.49 & 40.15 \\
\hline 5 & 80 & 18.12 & 24.56 & 26.92 & 46.44 & 28.85 & 45.96 \\
\hline 6 & 100 & 22.42 & 26.65 & 30.59 & 51.56 & 30.91 & 50.55 \\
\hline 7 & 120 & 23.59 & 27.05 & 34.49 & 55.69 & 33.18 & 54.72 \\
\hline 8 & 140 & 28.65 & 28.53 & 38.95 & 58.91 & 35.39 & 57.86 \\
\hline 9 & 160 & 31.95 & 29.75 & 43.65 & 60.08 & 37.98 & 60.98 \\
\hline 10 & 180 & 34.03 & 30.07 & 45.96 & 62.15 & 40.18 & 63.85 \\
\hline 11 & 200 & 36.95 & 33.05 & 47.56 & 63.95 & 42.59 & 65.97 \\
\hline 12 & 220 & 44.15 & 35.84 & 49.62 & 65.67 & 45.19 & 66.89 \\
\hline 13 & 240 & 47.36 & 37.71 & 52.25 & 67.89 & 46.15 & 67.56 \\
\hline
\end{tabular}

1/60/25:1 means catalyst amount in $\% \mathrm{v} / \mathrm{v}$, temperature in ${ }^{\circ} \mathrm{C}$ and methanol to oil molar ratio respectively

\begin{tabular}{llll}
\hline S. no. & Property & Determined in present study & $\begin{array}{l}\text { Standard value/range } \\
\text { Canoira et al. [36] }\end{array}$ \\
\hline 1 & Kinematic viscosity $\left(\mathrm{mm}^{2} / \mathrm{s}\right.$ at $\left.40{ }^{\circ} \mathrm{C}\right)$ & 18.55 & $3.5-5.00$ \\
2 & Density $\left(\mathrm{kg} / \mathrm{m}^{3}\right)$ & 920.5 & $860-900$ \\
3 & Acid Value $(\mathrm{mg} \mathrm{KOH} / \mathrm{g})$ & 0.25 & $<0.50$ \\
4 & Flash point $\left({ }^{\circ} \mathrm{C}\right)$ & 170 & $>120$ \\
5 & Water content $(\%)$ & 0.006 & $<0.0005$ or $<500 \mathrm{mg} / \mathrm{kg}$ \\
6 & Calorific value $(\mathrm{MJ} / \mathrm{kg})$ & 39.5 & $37.27-38.22$ \\
7 & Oxidation stability at $110{ }^{\circ} \mathrm{C}(\mathrm{h})$ & 8 & $>6.0$ \\
8 & Sulfur content $(\mathrm{mg} / \mathrm{kg})$ & 0.3 & $<10$ \\
\hline
\end{tabular}




\section{Modeling of experimental observations through ANN}

ANNs are useful for the study of complex phenomena for which we have appropriate data but a poor understanding of the mathematical relationship between them [37]. There are several modeling strategies in ANNs, which have various applications in designing and analyzing existing processes. Choosing the right network architecture is one of the most important tasks prior to the modeling process. A feed-forward neural network architecture was selected for the model development owing to its powerful non-linear mapping ability between inputs and outputs [38], which consisted of an input layer, an intermediate hidden layer and an output layer. In a typical feed-forward network every node in each layer is connected to all the nodes in the following layer. The neurons in the hidden layer often consist of sigmoidal neurons. A sigmoid function is a bounded differentiable real function that is defined for all real input values and has a positive derivative at each point. Tangent sigmoid function was preferred as the activation function in the hidden layer for all neural network computations.

$\tan \operatorname{sig}(x)=2 /(1+\exp (-2 x))-1$.

A total of 156 experimental datasets were used to train (130 datasets; columns A-J in Table 2a, b) and to test (26 datasets; columns $\mathrm{K}$ and $\mathrm{L}$ in Table $2 \mathrm{~b}$ ) the performance of the developed ANN. Each dataset includes four input variables namely, methanol to oil molar ratio (mr), catalyst amount $(c)$, temperature $(T)$ and time $(t)$. Scaling of the input and output variable is usually recommended [39]. Therefore, each input variable was scaled in the range $\{0$, $1\}$ by dividing them with their maximum values, respectively, in Table 4 . These scaled variables were applied to the input neurons to carry out the ANN modeling. The available data was distributed into three different sets for the training method, i.e., $70 \%$ for the training set, $15 \%$ for the validation set and remaining $15 \%$ for the test set.

The Levenberg-Marquardt algorithm works on the principle of error-back propagation, and was used to train the neural network [40]. Mean square error (MSE) was chosen as the performance function for observing

Table 4 Maximum values of input and output variables

\begin{tabular}{lll}
\hline S. no. & Variables & Maximum value \\
\hline 1 & Molar ratio (-) & $25: 1$ \\
2 & Catalyst amount $(\% \mathrm{v} / \mathrm{v})$ & 3.68 \\
3 & Temperature $\left({ }^{\circ} \mathrm{C}\right)$ & 60 \\
4 & Time $($ min $)$ & 240 \\
5 & FAME yield $(\%)$ & 100 \\
\hline
\end{tabular}

deviations between experimental data and calculated data from the network's output layer.

For the present study, MSE was given by:

MSE $=\sum_{k=1}^{N}\left(\frac{X_{k}^{\text {exp }}}{100}-\frac{X_{k}^{\text {cal }}}{100}\right)^{2} / N$,

where $N$ : number of datasets, $X_{k}^{\text {exp }}$ : experimental value of the FAME percentage yield, $X_{k}^{\text {cal }}$ : calculated value of the FAME percentage yield.

\section{Parameter estimation}

Parameter estimation has been carried out for the data obtained experimentally and others generated by using ANN model. Several physical and kinetic parameters appear in the process model developed for transesterification of castor oil. The physical parameters, like the reactor volume and species densities were fixed based on the process knowledge. Each kinetic parameter has a pre-exponential factor and activation energy associated with it. The activation energies $(E)$ have been fixed as the temperature $(T)$ and catalyst concentration was taken to be the same for all the molar ratios.

$k=k_{0} e^{\left(-\frac{E}{\mathrm{RT}}\right)}$,

where $k$ : reaction constant at given temperature $(T), k_{0}$ : reaction constant at reference temperature.

Various parameter estimation techniques such as maximum likelihood estimation [41], prediction error minimization (PEM) [42], trust-region SQP algorithm [43] etc. have been used previously by researchers to estimate parameters of a model. Further, Hosten and Emig [44] have developed sequential experimental design procedures for precise parameter estimation in ordinary differential equations. In this work, parameter estimation technique based on a prediction error minimization (PEM) framework has been used. A central point in the PEM approach is to design a predictor, which is a function that returns a predicted value of the output of the system for given parameter values and a sequence of measurements. Comparison of the predicted value with an actual measurement gives the prediction error, which can be seen as a function of the system parameters. The prediction errors are squared and summed together. This function minimization with respect to the parameters is a way of finding good parameter estimates. Similar works have been performed by Mjalli and Ibrehem [42] and Mallikarjunan et al. [45] where they have used optimal hybrid modeling approach for polymerization reactors using PEM technique.

In this work, the parameters values were estimated using MATLAB 7.6 (R2008a). The MATLAB code consisted of optimization tool 'fminsearch'. The differential equation was solved symbolically using 'dsolve' command. 
$X_{c_{i, j}}=g\left(t_{i, j}, k_{1}, k_{2}, C_{\mathrm{A}_{i}}, M_{i}\right)$.

The function which has been minimized is as follows:

$\sum_{i=1}^{N} \sum_{j=1}^{M} \times\left[X_{\mathrm{c}_{i, j}}-g\left(t_{i, j}, k_{1}, k_{2}, C_{\mathrm{A}_{i}}, M_{i}\right)\right]^{2}$,

where $X_{\mathrm{c}_{i, j}}$ : values obtained experimentally and predicted through ANN, $M$ : number of data points and $N$ : number of molar ratios.

The function 'fminsearch' finds the minimum of a scalar function of several variables, starting at an initial estimate. This is generally referred to as unconstrained nonlinear optimization. 'fminsearch' uses the simplex search method. This is a direct search method that does not require numerical or analytic gradients. If $n$ is the length of $x$, a simplex in $n$ dimensional space is characterized by the $n+1$ distinct vectors that are its vertices. In two-dimensional space, a simplex is a triangle; in three-dimensional spaces, it is a pyramid. At each step of the search, a new point in or near the current simplex is generated. The function value at the new point is compared with the function's values at the vertices of the simplex and, usually, one of the vertices corresponding to worst value of function is replaced by the new point, giving a new simplex. This step is repeated until the size of the simplex is less than the specified tolerance [46].

\section{Results and discussion}

\section{Training of the ANN network}

The feed-forward network was developed by training it with various number of combinations of sigmoidal neurons in one and two hidden layers. The MSE was calculated for all uni-layered architecture and a decreasing behavior was observed for the training MSE when hidden layer size was increased (Fig. 1a).

When hidden neurons are increased beyond a certain level, over fitting occurs and network adapts to the noisy training data [47]. Over fitting easily leads to the disturbance of the network and predictions often lie outside the range of considered variables in a multilayered network even if the input data are totally noise free [48].

Different weight initializations have been used to train to prevent the network from converging to a local minimum which gives erroneous results. The optimal value of the number of neurons employed in the single hidden layer was set at 12 beyond which training and testing MSE start to diverge uncontrollably on further increase in the number of hidden neurons. Since, we have four different scaled input variables and one variable (fractional formation of FAME) as our desired output, our chosen topology for the FF-ANN bears a 4-12-1 relationship (Fig. 2).
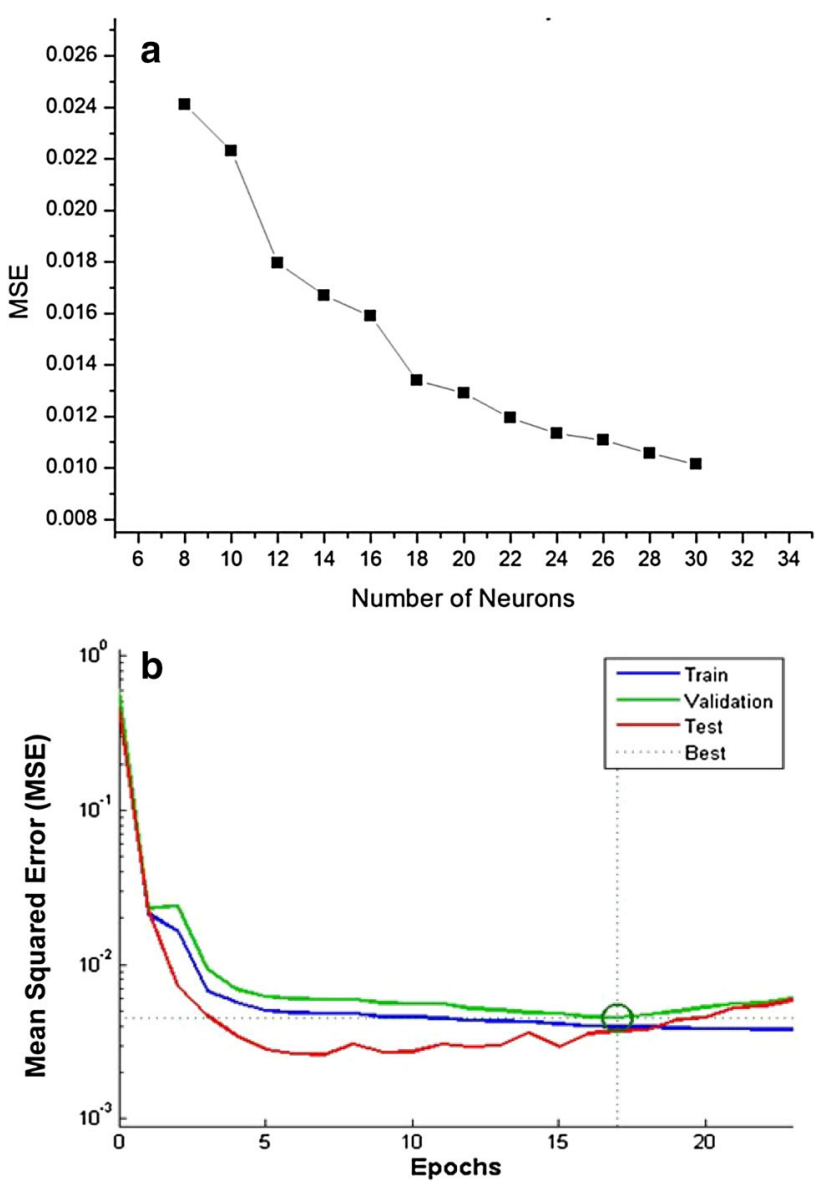

Fig. 1 a Training MSE behaviour for uni-layered topology. b Performance plot for the chosen architecture

Training of the chosen network was stopped when validation gradient started to overshoot. The best validation performance that gave the overall global minima of the system, was reported as 0.0045476 for the network at 17 th epoch during the training (one epoch describes the time it takes to train the network once using the back propagation algorithm), Fig. 1b. The desired outputs were obtained from the output layer of the network.

An overall regression coefficient of 0.953 was obtained for the network which showed that the network had been satisfactorily trained (Fig. 3). A few outliers were observed which are common in any neural network development due to various factors such as experimental error, observational error etc. This trained network was further used for the validation and testing procedures (Sects. 3.2, 3.3).

\section{ANN model validation}

The optimized ANN model was validated using two different sets of experimental data other than those used in developing ANN model having 13 data each. For this purpose, MATLAB 8.1(2013a) neural network toolbox [49] was 
Fig. 2 The 4-12-1 network topology used for modeling

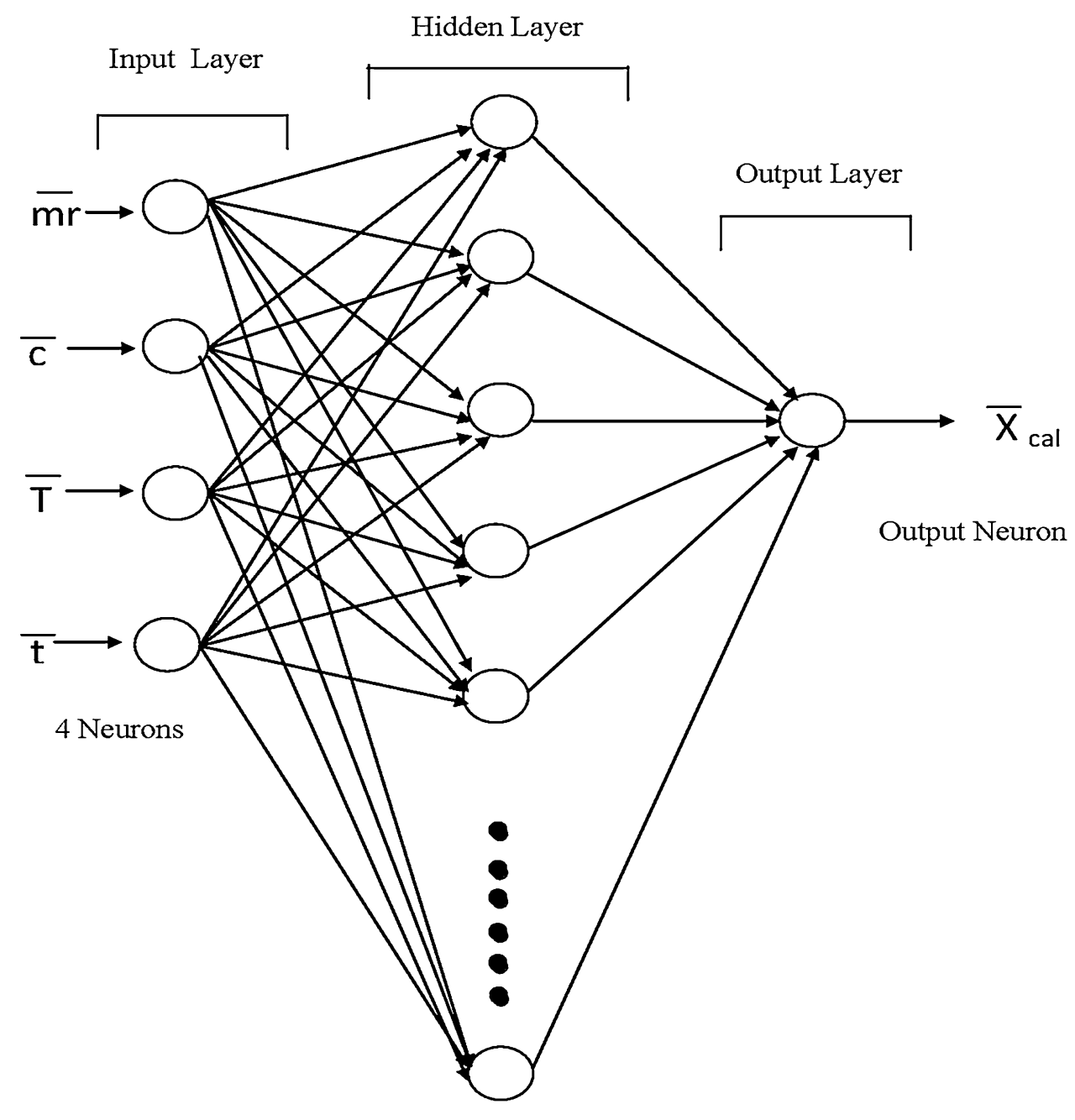

12 Neurons employed. These additional data were obtained by conducting experiments on the same lab reactor at 45 and $60{ }^{\circ} \mathrm{C}$, respectively (columns $\mathrm{K}$ and $\mathrm{L}$ in Table $2 \mathrm{~b}$ ). Comparison between ANN predictions and experimental data for these two separate validation sets show that all the model predictions are within $\pm 8 \%$ deviation. The three-layered FF-ANN with 12 hidden neurons (4-12-1) was capable of predicting the output of the process and yielded a very good approximation to the castor oil transesterification process.

From the above investigation, it may be concluded that the network accounts well for the variation in input variables and the developed ANN model can be successfully applied in predicting the FAME yield at conditions in the range of considered variables.

\section{Predictions using ANN model}

The ANN model which was trained and tested against experimental data (see Sects. 3.1, 3.2) was used to predict
FAME yields at different input datasets. It was considered appropriate to develop a kinetic model at optimized conditions, determined earlier for the lab reactor used [31]. Therefore, predictions were made using the developed ANN model at the conditions (catalyst $=3 \% \mathrm{v} / \mathrm{v}$, temperature $=60{ }^{\circ} \mathrm{C}$, time $=0-4 \mathrm{~h}$ ) to study the effect of molar ratio on FAME yield. Predictions for different molar ratios 9:1, 12:1, and 15:1 are shown in Fig. 4. The results obtained for these conditions from the ANN model have been used in the following Sect. (3.4) to develop the kinetic model.

\section{Kinetic modeling}

\section{General reaction model}

In the preceding section, profiles for fractional formation of FAME with time have been obtained using developed ANN model for methanol to oil molar ratios namely, 9:1, 
Fig. 3 Regression plots for the network for a training,

b validation, $\mathbf{c}$ test, $\mathbf{d}$ overall
Training: $\mathrm{R}=0.95517$

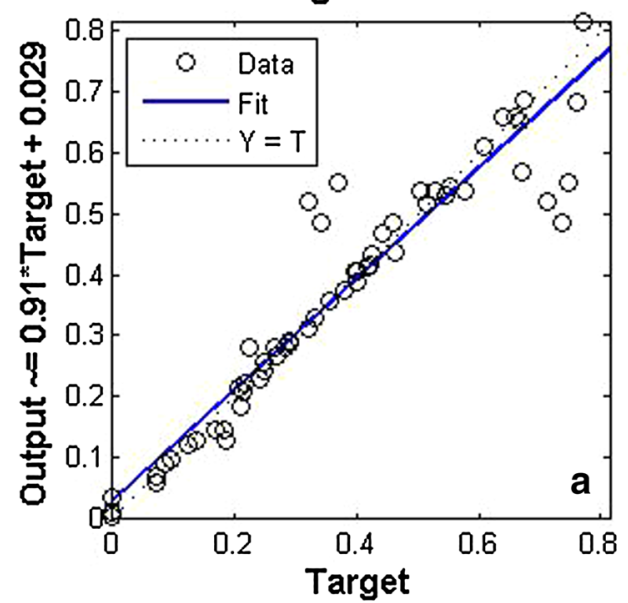

Test: $\mathbf{R}=0.94637$

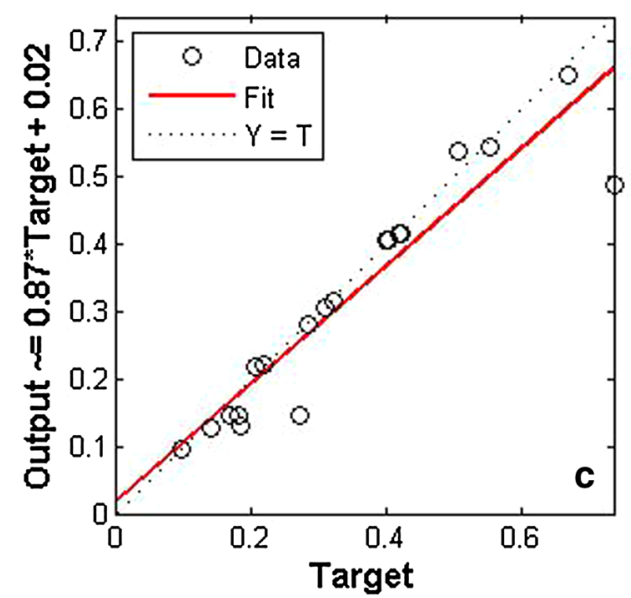

Validation: $\mathrm{R}=0.94986$

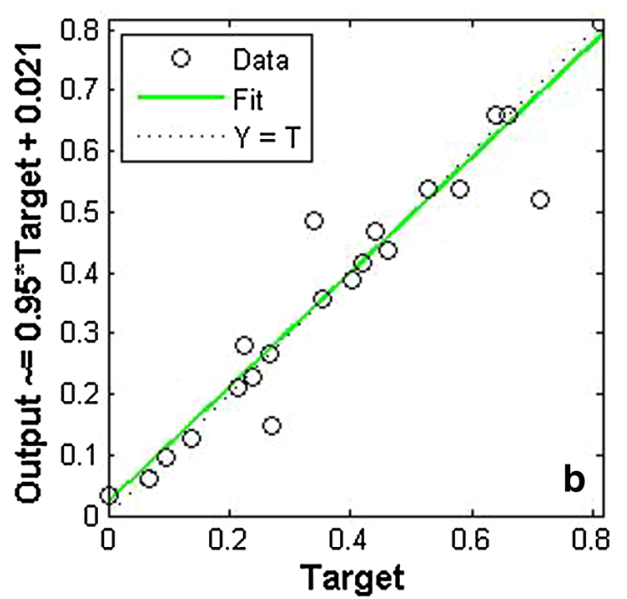

All: $\mathrm{R}=0.95267$

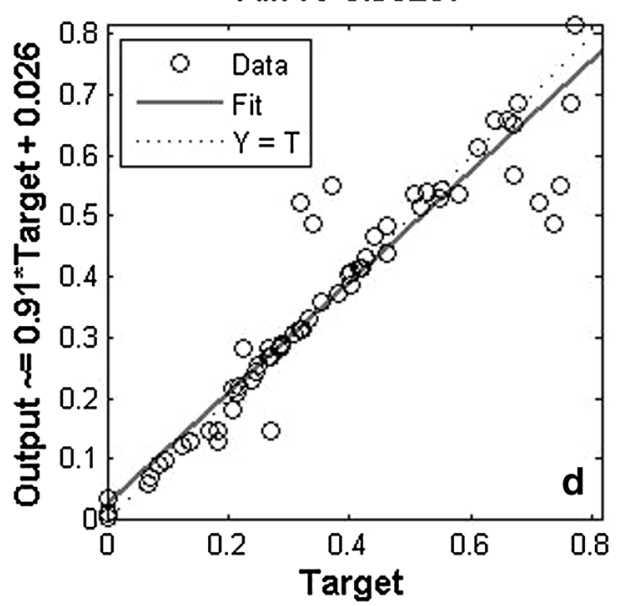

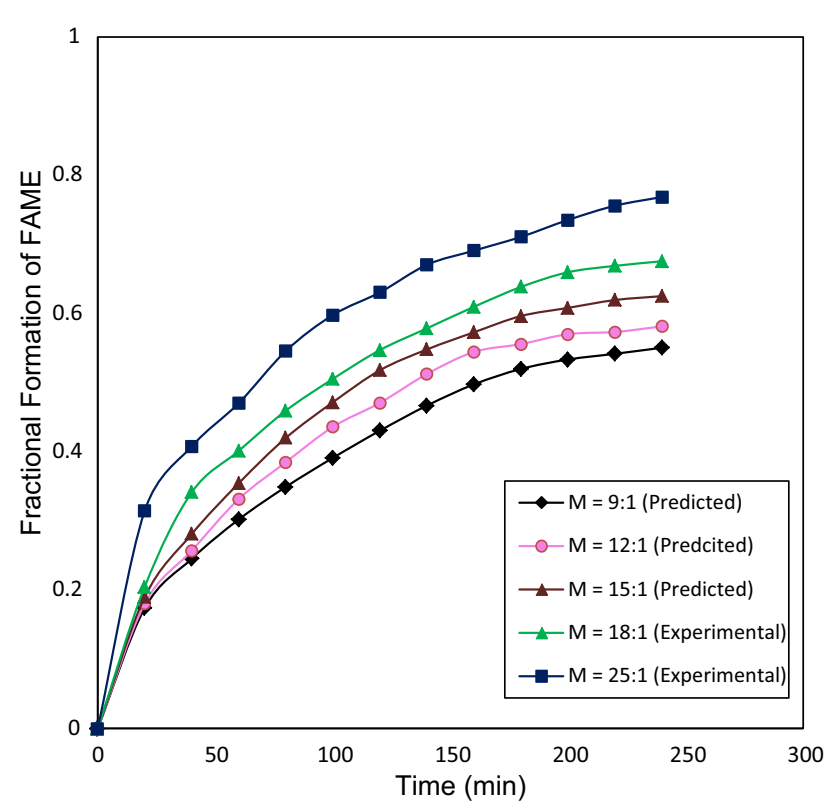

Fig. 4 ANN model predictions for intermediate molar ratios
$12: 1$, and $15: 1$, and experimental profiles for molar ratio 18:1 and 25:1 are already available at optimum catalyst amount and temperature. This section concerns the development of suitable kinetic model on the basis of these five profiles so that effect of change in molar ratio on fractional formation of FAME can be studied. The $C_{\mathrm{A} 0}$ values corresponding to different molar ratios used for fitting are given in the Table 5.

Following kinetic model was used to fit the data obtained by ANN. The kinetic model is in general reversible reaction model, in which both forward and backward reactions are second order, first order with respect to each of the reactants and products.

$\mathrm{d} X_{\mathrm{c}} / \mathrm{d} t=k_{1} C_{\mathrm{A} 0}\left(3-X_{\mathrm{c}}\right)\left(M-X_{\mathrm{c}}\right)-k_{2} C_{\mathrm{A} 0} X_{\mathrm{c}}^{2}$,

with $X_{\mathrm{c}}=0$ at $t=0$. Where $C_{\mathrm{A} 0}$ initial concentration of castor oil (mol/L), $X_{\mathrm{c}}$ : fractional formation of FAME, $M$ : molar ratio of methanol to castor oil, $k_{1}, k_{2}$ : rate constants (L $\mathrm{min} / \mathrm{mol}$ ). 
Table $5 C_{\mathrm{A} 0}$ values corresponding to different molar ratios

\begin{tabular}{llll}
\hline S. no. & Molar ratio $(\mathrm{M})$ & $C_{\mathrm{A} 0}(\mathrm{gmol} / \mathrm{L})$ & Catalyst $(\mathrm{vol} \% / \mathrm{vol})$ \\
\hline 1 & $9: 1$ & 0.733668 & $3 \%$ \\
2 & $12: 1$ & 0.67393 & $3 \%$ \\
3 & $15: 1$ & 0.622769 & $3 \%$ \\
4 & $18: 1$ & 0.57927 & $3 \%$ \\
5 & $25: 1$ & 0.49771 & $3 \%$ \\
\hline
\end{tabular}

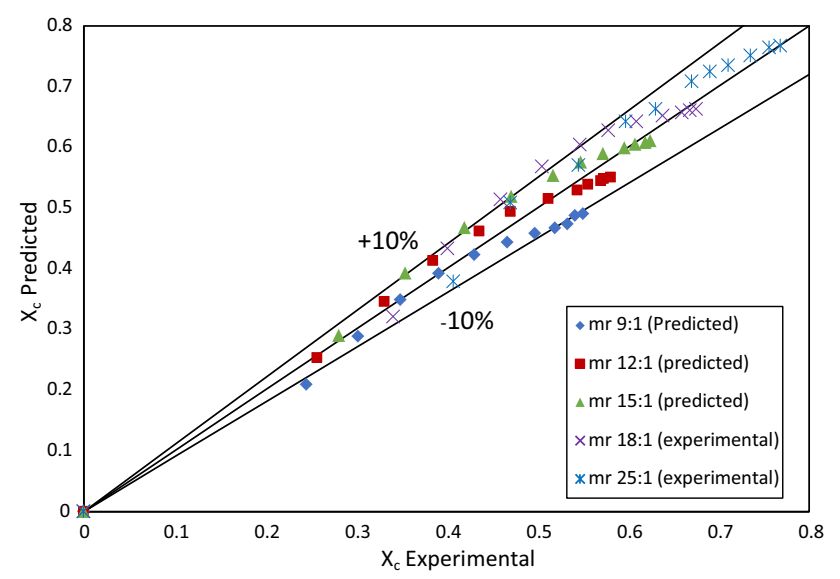

Fig. 5 Comparison of $X_{\mathrm{c}}$ predicted by kinetic model with $X_{\mathrm{c}}$ experimental

\section{Estimation of parameters}

The values of $k_{1}$ and $k_{2}$ were estimated using the procedure discussed in Sect. 2. The values estimated are $k_{1}=0.000262$ and $k_{2}=0.023178(\mathrm{~L} \mathrm{~min} / \mathrm{mol})$, respectively. The correlation coefficients and normalized \% standard deviations for different molar ratios i.e. 9:1, 12:1, 15:1, $18: 1$ and $25: 1$ were obtained as $(0.933453,8.783124 \%)$, $(0.989036, \quad 3.637967 \%), \quad(0.994593, \quad 2.760923 \%)$, $(0.983285,4.831601 \%)$, and $(0.974017,5.589103 \%)$, respectively. Higher values of correlation coefficient $(>0.93)$ and lower values of normalized \% standard deviation $(<9 \%)$ testify the goodness of fit of the kinetic model. To compare the accuracy of model, all the predicted values of fractional formation of FAME, $X_{\mathrm{c}}$ Predicted, by kinetic model, and its experimental values and generated values by ANN model, $X_{\mathrm{c}}$ experimental, have been plotted in Fig. 5 for all five molar ratios. From this figure, it is obvious that most of the predictions lie within $\pm 10 \%$ deviation.

\section{Conclusion}

In this research work, experiments have been conducted at 12 sets of operating conditions. A FF-ANN model (4-12-1) has been developed on the basis of these data. The model is capable of predicting fractional formation of FAME $\left(X_{\mathrm{c}}\right)$ at other operating conditions. In this work, $X_{\mathrm{c}}$ have been computed using the developed ANN model at 9:1, 12:1 and 15:1 methanol to oil molar ratio at $60^{\circ} \mathrm{C}$ and $3 \%$ catalyst loading. Further, a kinetic model has also been developed using the experimental and computed data at $60{ }^{\circ} \mathrm{C}$ and $3 \%$ catalyst loading. The kinetic model considers the transesterification reaction to be a second order reversible, first order with respect to each of the reactants and products, and is applicable to methanol to oil molar ratios varying between 9:1 and 25:1. Estimated values of kinetic constants $k_{1}$ and $k_{2}$ are 0.000262 and $0.023178 \mathrm{~L} \mathrm{~min} / \mathrm{mol}$, respectively.

Acknowledgements Authors are grateful to the Ministry of Human Resource Development (MHRD), Government of India, New Delhi for providing the financial assistance during the course of this research work.

Open Access This article is distributed under the terms of the Creative Commons Attribution 4.0 International License (http://crea tivecommons.org/licenses/by/4.0/), which permits unrestricted use, distribution, and reproduction in any medium, provided you give appropriate credit to the original author(s) and the source, provide a link to the Creative Commons license, and indicate if changes were made.

\section{References}

1. Oliveira FCC, Brandao CRR, Ramalho HF, da Costa LAF, Suarez PAZ, Rubim JC (2007) Adulteration of diesel/biodiesel blends by vegetable oil as determined by Fourier transform (FT) near infrared spectrometry and FT-Raman spectroscopy. Anal Chim Acta 587:194-199

2. Baptista P, Felizardo P, Menezes JC, Correia MJN (2008) Multivariate near infrared spectroscopy models for predicting the iodine value, CFPP, kinematic viscosity at $40 \mathrm{C}$ and density at 15 C of biodiesel. Talanta 77:144-151

3. Knothe G, Sharp CA, Ryan TW (2006) Exhaust emissions of biodiesel, petrodiesel, neat methyl esters, and alkanes in a new technology engine. Energy Fuel 20:403-408

4. Leung DYC, Wu X, Leung MKH (2010) A review on biodiesel production using catalyzed transesterification. Appl Energy 87:1083-1095

5. Porte AF, CdS Schneider Rd, Kaercher JA, Klamt RA, Schmatz WL, da Silva WLT (2010) Sunflower biodiesel production and application in family farms in Brazil. Fuel 89:3718-3724

6. Gomes MCS, Arroyo PA, Pereira NC (2011) Biodiesel production from degummed soybean oil and glycerol removal using ceramic membrane. J Membr Sci 378:453-4561

7. Xin J, Imahara H, Saka S (2008) Oxidation stability of biodiesel fuel as prepared by supercritical methanol. Fuel 87:1807-1813

8. Kansedo J, Lee KT, Bhatia S (2009) Cerbera odollam (sea mango) oil as a promising non-edible feedstock for biodiesel production. Fuel 88:1148-1150

9. Berman P, Nizri S, Wiesman Z (2011) Castor oil biodiesel and its blends as alternative fuel. Biomass Bioenergy 35:2861-2866

10. Hincapie H, Mondragon F, Lopez D (2011) Conventional and in situ transesterification of castor seed oil for biodiesel production. Fuel 90:1618-1623

11. Meneghetti SMP, Meneghetti MR, Wolf CR, Silva EC, Lima GES, Coimbra MDA, Soletti JI, Carvalho SHV (2006) 
Ethanolysis of castor and cottonseed oil: a systematic study using classical catalysts. JAOCS 83:819-822

12. Thomas TP, Birney DM, Auld DL (2012) Viscosity reduction of castor oil esters by the addition of diesel, safflower oil esters and additives. Ind Crops Prod 36:267-270

13. Pradhan S, Madankar CS, Mohanty P, Naik SN (2012) Optimization of reactive extraction of castor seed to produce biodiesel using response surface methodology. Fuel 97:848-855

14. Ramezani K, Rowshanzamir S, Eikani MH (2010) Castor oil transesterification reaction: a kinetic study and optimization of parameters. Energy 34:4142-4148

15. Lopez JM, Garcia Cota TDNJ, Monterrosas EEGR, Martineza N, de la Cruz Gonzalez VM, Flores JLA, Ortega YR (2011) Kinetic study by $1 \mathrm{H}$ nuclear magnetic resonance spectroscopy for biodiesel production from castor oil. Chem Eng J 178:391-397

16. Jeong GT, Park DH (2009) Optimization of biodiesel production from castor oil using response surface methodology. Appl Biochem Biotechnol 156:431-441

17. Cavalcante KSB, Penha MNC, Mendonça KKM, Louzeiro HC, Vasconcelos ACS, Maciel AP, de Souza AG, Silva FC (2010) Optimization of transesterification of castor oil with ethanol using a central composite rotatable design (CCRD). Fuel 89:1172-1176

18. Madankar CS, Pradhan S, Naik SN (2013) Parametric study of reactive extraction of castor seed (Ricinus communis L.) for methyl ester production and its potential use as bio lubricant. Ind Crops Prod 43:283-290

19. Tiwari AK, Kumar A, Raheman H (2007) Biodiesel production from jatropha oil (Jatropha curcas) with high free fatty acids: an optimized process. Biomass Bioenergy 31:569-575

20. Canakci M, Gerpen JV (2001) Biodiesel production from oils and fats with high free fatty acids. Trans ASAE 44:1429-1436

21. Boucher MB, Unker SA, Hawley KR, Wilhite BA, Stuart JD, Parnas RS (2008) Variables affecting homogeneous acid catalyst recoverability and reuse after esterification of concentrated omega- 9 polyunsaturated fatty acids in vegetable oil triglycerides. Green Chem 10:1331-1336

22. Soldi RA, Oliveira ARS, Ramos LP, Cesar-Oliveira MAF (2009) Soybean oil and beef tallow alcoholysis by acid heterogeneous catalysis. Appl Catal A Gen 361:42-48

23. Barton R (1995) Analysis and rectification of data from dynamic chemical processes via artificial neural networks. Ph. D Dissertation, The University of Texas at Austin, Austin, Texas

24. Suewatanakal W (1993) A comparison of fault detection and classification using ANN with traditional methods. Ph. D. Dissertation, The University of Texas at Austin, Austin, Texas

25. Karjala TW (1995) Dynamic data rectification via recurrent neural networks. Ph. D Dissertation, The University of Texas at Austin, Austin, Texas

26. MacMurray JC(1993) Modelling and control of a packed distillation column using artificial neural networks. M.S. Thesis, The University of Texas at Austin, Austin, Texas

27. Rajendra M, Jena PC, Raheman H (2009) Prediction of optimized pretreatment process parameters for biodiesel production using ANN and GA. Fuel 88:868-875

28. Daponte P, Grimaldi D (1998) Artificial neural networks in measurements. Measurement 23:93-115

29. Balabin RM, Lomakina EI, Safieva RZ (2011) Neural network (ANN) approach to biodiesel analysis: analysis of biodiesel density, kinematic viscosity, methanol and water contents using near infrared (NIR) spectroscopy. Fuel 90:2007-2015
30. Chakraborty R, Sahu H (2013) Intensification of biodiesel production from waste goat tallow using infrared radiation: process evaluation through response surface methodology and artificial neural network. Appl Energy 114:827-836

31. Payal PhD (2014) Thesis on castor oil transesterification: experimental and modelling studies. Indian Institute of Technology Roorkee, Roorkee

32. Lazíc ZR (2004) Design of experiments in chemical engineering. Wiley, Oxford

33. Yuan X, Liu J, Zeng G, Shi J, Tong J, Hunag G (2008) Optimization of conversion of waste rapeseed oil with high FFA to biodiesel using response surface methodology. Renew Energy 33:1678-1684

34. Kilic M, Uzun BB, Putun E, Putun AE (2013) Optimization of biodiesel production from castor oil using factorial design. Fuel Process Technol 111:105-110

35. Chaudhary P, Kumar B, Kumar S, Gupta VK (2015) Transesterification of castor oil with methanol-kinetic modeling. Chem Prod Process Model 10(2):71-80

36. Canoira L, Galean JG, Alcantara R, Lapuerta M, Contreras RG (2010) Fatty acid methyl esters (FAMEs) from castor oil: production process assessment and synergistic effects in its properties. Renew Energy 35:208-217

37. Gorr WL, Nagin D, Szczypula J (1994) Comparative study of artificial neural network and statistical models for predicting student grade point averages. Int J Forecast 10:17-34

38. Haykin S, Networks Neural (1999) A comprehensive foundation, 2nd edn. Prentice-Hall, Upper Saddle River

39. Hojjat H, Etemad SG, Bagheri R, Thibault J (2011) Thermal conductivity of non-Newtonian nanofluids: experimental data and modeling using neural network. Int J Heat Mass Transf 54:1017-1023

40. Hagan MT, Menhaj M (1994) Training feed forward networks with the Marquardt algorithm. IEEE Trans Neural Netw 5:989-993

41. Bindish R, Rawlings JB (2003) Parameter estimation for industrial polymerization processes. AIChE J 49:2071-2078

42. Mjalli FS, Ibrehem AS (2011) Optimal hybrid modelling approach for polymerization reactors using parameter estimation techniques. Chem Eng Res Des 89:1078-1087

43. Arora N, Biegler LT (2004) Parameter estimation for a polymerization reactor model with a composite-step trust-region NLP algorithm. Ind Eng Chem Res 43:3616-3631

44. Hosten LH, Emig G (1975) Sequential experimental design procedures for precise parameter estimation in ordinary differential equations. Chem Eng Sci 30:1357-1364

45. Mallikarjunan V, Pushpavanam S, Immanuel CD (2010) Parameter estimation strategies in batch emulsion polymerization. Chem Eng Sci 65:4967-4982

46. Lagarias JC, Reeds JA, Wright MH, Wright PE (1998) Convergence properties of the Nelder-Mead simplex method in low dimensions. SIAM J Optim 9(1):112-147

47. Cavas L, Karabay Z, Alyuruk H, Dogan H, Demir GK (2011) Thomas and artificial network models for the fixed-bed adsorption of methylene blue by a beach waste Posidonia oceanic (L.) dead leaves. Chem Eng J 171:557-562

48. Himmelblau DM (2008) Accounts of experiences in the application of artificial neural networks in chemical engineering. Ind Eng Chem Res 47:5782-5796

49. Beale M, Hagan M, Demuth H (2013) MATLAB Neural network toolbox user's guide (R2013A) 